\author{
Cosmin GHEȚĂU* \\ Mihai-Bogdan IOVU**
}

\title{
I Hear You: Effects of Voice Communication on the Social Capital of Gamers
}

\begin{abstract}
In the present paper we approached bonding and bridging social capital among gamers (seen as individuals who spend at least an hour a day in games) who use (or don't use) voice communication while playing. Suspecting that voice communication usage facilitates the social capital formation. We also investigated the role of perceived anonymity as a confounding variable. To test our hypothesis, a quantitative research was carried out. The study focuses on the subsequent elements: voice application usage; perceived anonymity; and the presence of the following forms of social capital: bonding social capital and bridging social capital. Data collection was based on surveys spread in the online environment and resulted in a sample of 102 respondents. Based on quasi-experimental design we obtained data that display higher mean scores on bonding and bridging social capital scales on the experiment group (voice communication users) than in control group (non-voice communication users). Also, control group have a lower score on the perceived anonymity scale. One explanation for our results could be that voice communication reduces perceived anonymity and individuals that consider themselves as having a low level of anonymity engage in more controlled behaviors that in turn facilitate the formation of social capital.
\end{abstract}

Key words: Social Capital; Video Games; Voice Communication; Perceived Anonymity; Gaming;

\section{Introduction}

Video games are increasing their popularity consolidating their position as the most chosen leisure activity (Deleuze, et al., 2019). In 2019 Entertainment Software Association reports $65 \%$ of adults from United States that are supposedly video game players and according to the Interactive Software Federation of Europe in 2020 over 51\% of the population aged 664 from Europe play video games among which 45\% of game players are women. In 2021 it is estimated that over 2.8 billion persons play video games worldwide that generate revenues of $\$ 189.3$ billion to the gaming market (Newzoo, 2021). This popularity has driven the interest of researchers from different fields. Studies show that video games present advan-

* Babeș Bolyai University (Romania) cosmin.ghetau@ubbcluj.ro

** Babeș Bolyai University (Romania) mihai.iovu@ubbcluj.ro 
tages such as: improving concentration, working memory and even multitasking abilities (von der Heiden, Braun, Muller, \& Egloff, 2019), improving cognitive abilities (Dobrowolski, Hanusz, Sobczyk, Skorko, \& Wiatrow, 2015) and in case of online games, improved social skills (Steinkuehler \& Williams, 2006; Huvila, Holmberg, Ek, \& Widen, 2010; Trepte, Reinecke, \& Juechems, 2012; Kobayashi, 2010; Molyneux, Vasudevan, \& Gil de Zúñiga, 2015). Previous studies have led many whom are interested in the social aspects of video games to be interested in the mechanism that can enhance the potential social benefits of gaming.

One way of investigating social aspects within games is based on the Social Capital theory. This form of capital is understood as the sum of all the resources "an actor can mobilize and/or profit from/because of his embeddedness in a network of relations with other actors" (Castiglione, Van Deth, \& Wolleb, 2008, p. 23).

Although there are studies asserting the effect of video games, especially online ones on Social Capital formation and its maintenance (Steinkuehler \& Williams, 2006; Huvila, Holmberg, Ek, \& Widen, 2010; Trepte, Reinecke, \& Juechems, 2012; Kobayashi, 2010; Molyneux, Vasudevan, \& Gil de Zúñiga, 2015), not as many address the effect on the Social Capital exercised by the increasing popularity of the voice chat applications dedicated to gaming, e.g. Discord.

The development of these applications is driven by the same reasons the social researchers have for leaning more and more toward video games: the increasing number of people playing online games. In these games, the participants "meet" with friends, family, or even strangers with whom they have no connection, crossing wide geographical distances and cultural borders (Cole \& Griffiths, 2007; Granic, Lobel, \& Engels, 2014). Thus, this reduces the differences between generations, socio-economic status, and language barriers (Granic, Lobel, \& Engels, 2014). Correspondingly, a large spectrum of social issues is found in online games, or more precisely in games that fit into the Massively Multiplayer Online (MMO) genre. The MMO genre is composed, as the name suggest, by games with a large number of active players (Lucas \& Sherry, 2004; Krotoski, 2004; Cole \& Griffiths, 2007). In this type of games, players can interact with others throughout the game network and establish new friendships or even romantic relationships, through computer-mediated and game-offered communication methods (Lucas \& Sherry, 2004).

Studies show that social interactions in online games, especially MMOs, form a considerable drive-in video game preference and contributes to the well-being of the player (Cole \& Griffiths, 2007; Yee, 2006; Vella, Johnson, \& Hides, 2013). MMO games have in-game builtin tools that facilitate the development of long-lasting online communities, such as the guild systems (Ducheneaut, Yee, Nickell, \& Moore, 2007; Nardi, 2010). Guilds are communities with a clear social structure, with a "leader" and "officers" and sometimes ranks that members can reach (Nardi, 2010, p. 15).

But by far, the most important element of this kind of games is communication, which can be considered the main element that keeps the game alive. For this reason, online games have their own mechanisms aimed at facilitating discussion or chat between players. Steinkuehler \& Duncan (2008) conducted a study on these mechanisms, specifically on discussion threads (public written conversations) in a multiplayer online game, and found that most discussions focused on solving common problems, including sharing knowledge.

However, players were not satisfied with these tools accessible in the game. Thus, with the growth of the community, new communication tools (external to the game) become popular among gamers which were dissatisfied with restriction related to texting (that request at- 
tention shift from game action to the keyboard) and turn to voice communication (Wadley, Gibbs, Hew, \& Graham, 2003).

As a result, new applications that focused on gamers communication have been developed, a good example being Discord, an application build from scratch to facilitate gaming communication. This app reached a record of 250 million users in just four years after its launch (Handrahan, 2019).

Regardless of the application gamers use to voice chat, the group connected to the conversation have at least one unique element, all users share one common interest: the video game they are playing and most probably video games in general. With all these aspects in mind, we could suspect a stronger connection between voice communication users. And, therefore, we hypothesize that voice communication will facilitate more the formation of the Social Capital compared to traditional online communication methods (e.g. mail, chat rooms, forums). Because of the game flow, using on text-based communication methods makes it difficult to chat and concentrate on the game (or at game commands) at the same time (Wadley, Gibbs, Hew, \& Graham, 2003). Having their eyes on the game and hands on commands, players could small talk other things, such as what they like in the game or even details about personal life, as the game progress (Nardi, 2010).

\subsection{Why players would use voice communication?}

The success of voice communication applications (such as Discord) may be based on the fact that online video games are developed from scratch to be played in groups or are more entertaining when players play in groups (Williams, Caplan, \& Xiong, 2007). Two of the most popular types of games at the moment (Team-based Multiplayer First-Person Shooter: FPS and Multiplayer online battle arena: MOBA) have as main element: „team work”. In League of Legends (MOBA) a player must work with four other players to bring down the base of the enemy team (also composed by five players). In Overwatch (Team-based Multiplayer FPS), the team is bigger (two teams of six players), yet the purpose is the same: the player with his teammates need to fight another team composed of an equal number of members.

As games require maintaining a constant communication flow but also focusing on game controls, players have increasingly considered voice chat an alternative as this form of communication does not have the same restrictions as text chat (Wadley, Gibbs, Hew, \& Graham, 2003).

Even in games where the player does not necessarily need to fight against other real people, online games still emphasize on group play. An example could be the "raids", specific to the Massively Multiplayer Online Role-Playing Games (MMORPGs). Raids (mechanisms) can be explained as "the conquest of a castle": players need to gather in a group to defeat the powerful virtual opponents created and controlled by the game (depending on the game, these groups consist of: 5, 10, 25 or even more players). Williams et al. (2006) describes raids as "the most complex team-based task within MMOs and requires a certain amount of individual discipline and teamwork" (Williams, et al., 2006, p. 345) . The action in the raid is in a "constant dynamic change" (Nardi, 2010, p. 55), and in order to function well, each player must act in the interest of the group, "requiring a high degree of familiarity and practice" (Williams, et al., 2006, p. 345). Thus, these groups "nearly always use voice chat" (Nardi, 2010, p. 9). 


\subsection{Forms of Social Capital}

Research on social interaction and friendship formation has differentiated between two types of social bonds: Bridging Social Capital and Bonding Social Capital (Putnam, 2000).

"Bridging Social Capital" occurs when individuals from different backgrounds make connections through social networks. This form of Social Capital is marked by tentative relationships, but even if it provides little emotional support, such relationships can broaden a person's social horizons or perceptions, providing access to new information and resources (Putnam, 2000; Williams D. , 2006).

"Bonding Social Capital" refers to strong social bonds, because it occurs only between people who are united by strong bonds, such as family and close friends, relationships that provide emotional and background support (Steinkuehler \& Williams, 2006; Huvila, Holmberg, Ek, \& Widen, 2010; Putnam, 2000). Even the optimistic work of Cole and Griffiths (2007), identify a low level of strong relationships or relationships that materialize in offline relationships. Even so, it is possible that the intense use of voice communications among gamers in the last period has increased at least the familiarity between players (Nardi, 2010). Therefore, the presence of Bonding Social Capital cannot be completely ruled out from our assumptions.

\subsection{Anonymity in online games}

As the online world drive to connectivity (Sharon \& John, 2018) and the internet becomes constant in our lives "true anonymity is difficult, if not impossible, to acquire" (Hite, Voelker, \& Robertson, 2014, p. 22). The lack of absolute anonymity also has perverse effects. Reduced anonymity is shown as one important risk factor for future cyberbullying behavior (Barlett, Gentile, \& Chew, 2016, p. 179). Increased anonymity seems to promote undisciplined content on internet such as trolling, bulling and insults (Curlew, 2019; Sharon \& John, 2018). On the other hand, reduction in anonymity in a group can enhance decision quality, increase the quantity of ideas and solutions, or even increase the satisfaction of participants (Tsikerdekis, 2013). Anonymity was a strong element of gaming, where avatars that represent players rarely have the player real name or any personal information of the person behind the screen. The advantages and disadvantages of anonymity in online gaming have only recently begun to take on significance as players start to expose in games more than a mere avatar. Games become more socially integrated and many, especially the online games, have integrated some kind of community formation tools. Game communities that offer members a chance to share personal information's if they wish to do so (Nardi, 2010).

Correspondingly, the increasing popularity of voice communication also raises some privacy concerns. Voice communication, although useful in solving game tasks, affects the social sphere of the community. It also affects anonymity, by exposing the gender and sometimes even the age of the user (Nardi, 2010).

There is evidence that the perception of anonymity affects the behavior of players (Hite, Voelker, \& Robertson, 2014; Sticca \& Perren, 2013). A strong anonymity can have negative effects (e.g. can encourage cyberbullying) (Sticca \& Perren, 2013). On the other hand, internet anonymity allows people to discuss personal issues with strangers without worrying about disclosure of their real identity that in time is transpose in social trust (Zhai, 2019; Chen, Sun, $\mathrm{Wu}, \&$ Song, 2019). Sufficient level of trust may promote identity disclosure (Lawrence \& Grotz, 1977) and this identity disclosure is favorable to the development of social capital 
(Millen \& Patterson, 2003). So, perhaps, voice communication in games also affects the way players share information and in time how they perceived their anonymity in these virtual worlds that turn their behavior.

\section{Methods}

\subsection{Aim}

Our study aims to investigate the relationship between voice communication and the development of certain forms of Social Capital (SC). Supposedly voice communication usage facilitates SC formation. One way through which voice communication could increase social capital is through the reduction of Perceived Anonymity (PA) between gamers.

Trust and identity disclosure on the internet are similar to the results presented in the previous literature regarding anonymity (Hite, Voelker, \& Robertson, 2014; Zhai , 2019; Lawrence \& Grotz, 1977; Millen \& Patterson, 2003). Users that are initially anonyms may become more accommodate with each other as they use voice chat. This accommodation encouraging identity disclosure and as result a loss in anonymity that in turn promote positive behavior which increase social capital.

To investigate the hypothesis, we examined three variables: Voice communication usage, Perceived Anonymity and Online Social Capital, namely Online Bridging SC and Online Bonding SC.

Figure 1. Voice communication usage decrease Perceived Anonymity (PA) that in turn increase Social Capital (SC).

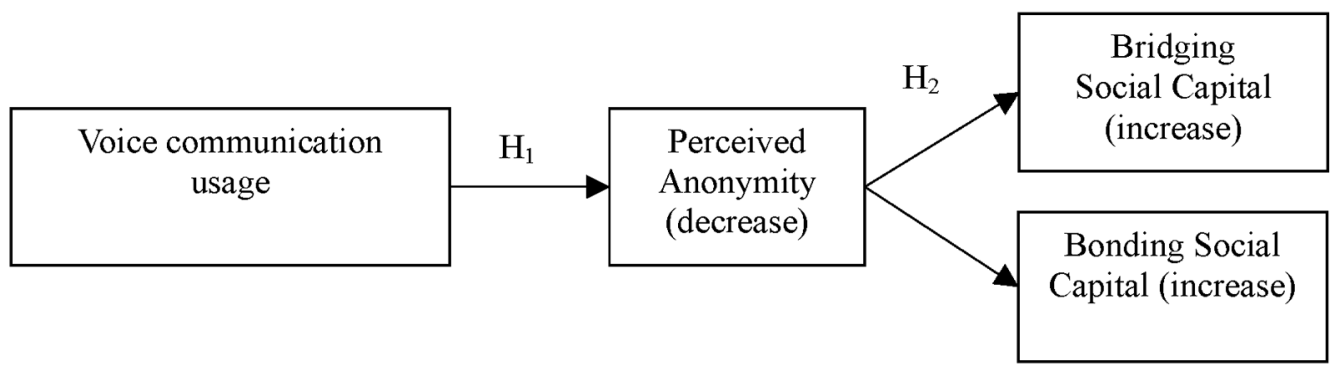

$H_{1}$ : Voice communication usage has a negative relationship with $P A$.

$\mathrm{H}_{2}:$ PA has a negative relationship with $\mathrm{SC}$.

\subsection{Measures}

Voice communication usage wile gaming

Usage of voice application was measured by a singular item (How often you use voice communication while playing) on a scale ranging from 1 to $10(1=$ not at all, $10=$ all the time). Upon completion, this was recoded into a dichotomous variable: Non-users (scores below 5) and Users (scores higher than 5). 


\section{Gamers Perceived Anonymity}

To measure anonymity of the responds we used the Perceived Anonymity Scale $(\mathrm{M}=2.6$, $\mathrm{SD}=1.1)(\mathrm{PA}$ : Hite, Voelker \& Robertson, 2014). This is a 5-item scale measuring the feeling of anonymity a person has in a specific situation. The items were scored on a 7-point Likert scale ranging from (1) strongly disagree to (7) strongly agree. According to Hite, Voelker, $\&$ Robertson (2014) the PA measure has good internal consistency reliability $(\alpha=.82)$.

\section{Online Social capital}

Social Capital was measured using Online Internet Social Capital Scales (ISCS). The original scale contains 40 items, of which 20 refer to online capital and 20 to offline capital. Each part can again be divided into two segments that distinguish between two forms of SC, namely bonding and bridging capital. We chose to use this scale precisely because of the possibilities of distinguishing several forms of SC. ISCS is one of the most used scale in measuring SC (Appel, et al., 2014) and its reliability on our target group has been confirmed in previous studies focusing on video game community (Williams, et al., 2006; Zhong, 2011; Trepte, Reinecke, \& Juechems, 2012).

Online Bridging Social Capital $(M=3.8, S D=.97)$ is a 10-item scale of the Internet Social Capital Scales (ISCS) developed by Williams (2006) that measures the presence as well as the intensity of bridging social capital (Putnam, 2000) in the online environment. The items are scored on a 5-point Likert scale ranging from (1) strongly disagree to (5) strongly agree.

Online Bonding Social Capital $(M=3.1, S D=1.0)$ is a 10-item scale the Internet Social Capital Scales (ISCS) developed by Williams (2006) measuring the presence as well as the intensity of bonding social capital (Putnam, 2000) in the online environment. The items are scored on a 5-point Likert scale ranging from (1) strongly disagree to (5) strongly agree.

According to Williams (2006) both scales used in the current study are valid and reliable, the scale author noted in the original paper forms and factor loadings for online versions: Bonding Factor, alpha $=.896$; Bridging Factor, alpha $=.841$.

\section{Socio-Demographic}

The Socio-Demographic items collected information on the following variables: gender $(0$ - male, 1 - female), age ( $1<14$ years, $2<18$ years, $3<25$ years old, $4<35$ years old, 5 $<45$ years old, $6<55$ years old), education (last form of education completed; $1-$ Elementary School, 2 - High School, 3 - Bachelor Degree, 4 - Master Degree, 5 - Doctoral Studies (PhD.), country (currently living country), residency, and gaming platform.

\subsection{Data collection}

Data collection was carried exclusively online. Our instrument was published once a week on several communities from social network sites (e.g., Reddit and Facebook) whose main topic of interest was video games (e.g., subreddits like r/videogame and r/gaming also Facebook groups like: Gaming Community and Global Gaming Guild, All for One Gaming, All For Gaming).

The questionnaire was written in English as we consider this the main language in which the respondents communicate online. In addition, writing in English allowed us to maintain the original items imported from other studies. 


\subsection{Sample}

The target population for this study was video games enthusiasts (named in the preset paper "gamers") that use voice communication while playing games and who spend at least one hour a day playing video games. Upon data collection completions we reach a sample of 102 respondents.

Over half of the sample is composed from young adults or youngster as the 18 to 24 years old group was the most numerous ( $\mathrm{N}=62,60.8 \%$ ) followed by the 25 to 34 years old group $(\mathrm{N}=30,29.4 \%)$, and 14 to 17 years old group $(\mathrm{N}=8,7.8 \%)$. There is also a discrepancy between respondents' gender. In our sample boys $(\mathrm{N}=65,63.7 \%)$ were overrepresented. Geographically, the majority was living at the moment in Romania ( $\mathrm{N}=79,77.5 \%)$, followed by United States $(\mathrm{N}=7,6.9 \%)$ and United Kingdom $(\mathrm{N}=5,4.9 \%)$. We also have at least one respondent from the following countries: Canada, India, Russia, Japan, Germany and Pakistan.

The most popular gaming devices used were: Personal Computers, including laptops (mean $=8.41 ; \mathrm{SD}=2.30$ ), Smartphones ( $m e a n=4.94 ; \mathrm{SD}=3.02)$, Gaming Consoles such as PlayStation $($ mean $=3.04 ; \mathrm{SD}=2.83$ ) and Mobile Consoles such as Nintendo Switch (mean $=2.28$; $\mathrm{SD}=2.62)$.

\section{Results}

In regard to voice application users, more than half of the sample $(\mathrm{N}=65,63.7 \%)$ are considered users and were allocated to the experimental group. The remaining respondents $(\mathrm{N}=$ $37,33.6 \%$ ) were allocated to the control group and were considered non-users (selected 5 or lower scores on the 1 to 10 liked scale on voice application usage).

Independent-samples t-test was run to compare the means differences in Online Social Capital (bridging and bonding) and in Perceived Anonymity Scale for the two testing conditions. There was a significant difference between users and non-users for Bridging Online Social Capital [ $\mathrm{t}(100=-3.07, \mathrm{p}=.003]$ with users displaying more Bridging $\mathrm{SC}(\mathrm{M}=3.93$, $\mathrm{SD}=.74)$ compared to the non-users $(\mathrm{M}=3.34, \mathrm{SD}=1.20)$. Similarly, there was a significant difference between the two groups when measuring Bonding Online Social Capital $[\mathrm{t}(100)=$ $-4.11, \mathrm{p}=.000]$ for users $(\mathrm{M}=3.28, \mathrm{SD}=.87)$ and non-users $(\mathrm{M}=2.43, \mathrm{SD}=1.22)$. Lastly, there also was a significant difference between the two groups when measuring Perceived Anonymity $[\mathrm{t}(100)=6.34, \mathrm{p}=.000$. $]$ for users $(\mathrm{M}=2.30, \mathrm{SD}=.98)$ and non-users $(\mathrm{M}=3.58$, $\mathrm{SD}=.97)$.

In addressing the effects between our investigated variables, correlational analysis was run. Results (see Table 1) show positive relations between Bridging Social Capital Scale and experimental groups $[\mathrm{r}(96)=.220, \mathrm{p}<.05]$, between Bonding Social Capital Scale and Experimental groups $[\mathrm{r}(96)=.343, \mathrm{p}<.01]$ and between the ISCS Online Mean (the two scales taken together) and Experimental Group $[\mathrm{r}(96)=.319, \mathrm{p}<.01]$. Also a negative correlation coefficient between the experimental groups and the anonymity scale $[\mathrm{r}(96)=-.511, \mathrm{p}<.001]$ has been found. There are negative coefficients between Perceived Anonymity Scale and the two scales of Social Capital calculated separately as distinct scales (Bonding SC Scale and Bridging SC Scale) and together as mean score (ISCS Online Scale). 
Table 1. Correlational analyses between investigated variables

\begin{tabular}{|c|c|c|c|c|c|c|c|}
\hline \multicolumn{3}{|c|}{ Correlations } & & & & & \\
\hline \multicolumn{3}{|c|}{ Control Variables } & $\begin{array}{c}\text { Experimental } \\
\text { Groups* }\end{array}$ & $\begin{array}{c}\text { ISCS } \\
\text { Bridging } \\
\text { Social } \\
\text { Capital } \\
\text { Scale }\end{array}$ & $\begin{array}{c}\text { ISCS } \\
\text { Bonding } \\
\text { Social } \\
\text { Capital } \\
\text { Scale }\end{array}$ & $\begin{array}{l}\text { ISCS } \\
\text { Online } \\
\text { Mean }\end{array}$ & $\begin{array}{l}\text { Perceived } \\
\text { Anonymity } \\
\text { Scale (PA) }\end{array}$ \\
\hline \multirow{15}{*}{$\begin{array}{l}\text { Age \& } \\
\text { Gender \& } \\
\text { Education } \\
\text { \& Country }\end{array}$} & \multirow{3}{*}{$\begin{array}{l}\text { Experimental } \\
\text { Groups }^{*}\end{array}$} & Correlation & & .220 & .343 & .319 & -.511 \\
\hline & & & & .030 & .001 & .001 & .000 \\
\hline & & df & & 96 & 96 & 96 & 96 \\
\hline & \multirow{3}{*}{$\begin{array}{l}\text { ICSC } \\
\text { Bridging }\end{array}$} & Correlation & & & .600 & .880 & -.310 \\
\hline & & & & & .000 & .000 & .002 \\
\hline & & df & & & 96 & .96 & 96 \\
\hline & \multirow{3}{*}{$\begin{array}{l}\text { ICSC } \\
\text { Bonding }\end{array}$} & Correlation & & & & .908 & -.541 \\
\hline & & & & & & .000 & .000 \\
\hline & & df & & & & 96 & 96 \\
\hline & \multirow{3}{*}{$\begin{array}{l}\text { ISCS Online } \\
\text { Mean }\end{array}$} & Correlation & & & & & -484 \\
\hline & & & & & & & .000 \\
\hline & & df & & & & & 96 \\
\hline & \multirow[t]{3}{*}{ PA } & & & & & & \\
\hline & & df & & & & & \\
\hline & & df & & & & & \\
\hline
\end{tabular}

* Dummy variable: $0=$ control group or non-users; $1=$ experimental group or users

Source: Data generated by the author

Simple linear regressions were performed to predict the effects of Voice Communication on Online SC seen as ISCS Online Mean (calculated as mean of the two components form Williams ISCS scale) but also the distinct two online ISCS Scales and voice communication usage (as a scale not spread in experimental group). Additionally, Voice Communication Usage effects on Perceived Anonymity were being regressed as well. Results of the analysis are displayed in Table 2. 
Table 2. Stepwise regression models on voice communication usage and the investigated types of social capital: ISCS Online Bridging and ISSC Online Bonding; mean score of the two distinct ISCS scales.

\begin{tabular}{|l|c|c|c|c|c|}
\hline DV: ISCS Online Bonding & $\mathrm{B}$ & $\mathrm{SE}$ & $\mathrm{B}$ & $\mathrm{t}$ & $\mathrm{Sig}$ \\
\hline Voice Communication Usage & .133 & .030 & .403 & 4.397 & .000 \\
\hline & & & & & \\
\hline DV: ISCS Online Bridging & & & & & \\
\hline Voice Communication Usage & .103 & .028 & .348 & 3.710 & .000 \\
\hline & & & & & \\
\hline DV: ISCS Online Mean & & & & & \\
\hline Voice Communication Usage & .118 & .026 & .419 & 4.618 & .000 \\
\hline & & & & & \\
\hline DV: Perceived Anonymity Scale (PA) & & & & & \\
\hline Voice Communication Usage & -.199 & .029 & -.566 & 19.383 & .000 \\
\hline & & & & & \\
\hline
\end{tabular}

Source: Data generated by the author

\section{Discussion and conclusions}

Similar to the data found in literature (Entertainment Software Association, 2019; Interactive Software Federation of Europe, 2020), we observed an increasing tendency in the mean age of gamers, also a greater number of girls that play video games.

Most of our respondents came from Romania. This may be due to the easier access to national social network groups. Also, members of the groups might have recognized the institutional affiliation of the researcher as trustworthy, therefore, they might have been more open to fill in the questionnaire.

The experimental group was slightly bigger than the control one (those that use more rarely speech to communicate). This was expected as voice communication between players becomes a norm (Nardi, 2010; Wadley, Gibbs, Hew, \& Graham, 2003).

Our study offers evidence in support of our hypothesis. Voice communication usage seem to facilitate the development of Social Capital (SC); experimental group present higher means on the ISCS Online Scale developed by Williams (2006). The aforementioned scale distinguish between two types of SC being composed of two distinct scales, one for Bonding Online SC and one for Bridging Online SC. In view of these peculiarities of the scale we were able to identify the relationships between these two distinct types of capital and the other variables investigated. Indeed, there seem to be some differences as the scores from Bonding SC have more significant positive correlation with voice communication users than Bridging.

Furthermore, Perceived Anonymity Scale scores are lower (and present a significant negative correlation) among voice communication users compared to non-users, thus indicating that voice communication is associated to perceived anonymity. Those who use voice communication perceive themselves as less anonym to their in-game peers that those that does not use voice. 
One explanation for our results could be that voice communication by itself influences perceived anonymity and individuals that consider themselves as having a low level of anonymity according to the literature, engage in more controlled behaviors (Hite, Voelker, \& Robertson, 2014; Sticca \& Perren, 2013) that in turn facilitate the development of SC (Zhai , 2019; Millen $\&$ Patterson, 2003).

We also might take into consideration that generally, players prefer to stay in touch with those they have played with before, so often the groups of regular players become in short time communities (Nardi, 2010). We suspect that players may prefer to keep in touch with those with whom have previously played for two reasons. One is a practical reason: it is easier to continue the game with people with whom you played in the past and thus learn the mechanisms together than with people who are either lower or higher in level (in game progress). The second reason is a personal/social one, they may have already settled in with those who have played together in the past and if they understand each other well in the past why not continue playing together.

Even though our data shows that voice communication has effects on SC (especially on the Bonding) and on perceived anonymity of gamers, we cannot be sure of the direction in which the variables influence each other. There might be other confounding variables that have not been identified (or addressed). To state with confidence the variables direction, longitudinal surveys have to be conducted. As voice communication becomes more of a norm, we consider these times as the perfect moment to conduct this kind of research, as in the future it may become difficult to recruit non voice communication users.

Limitations of the present study regard the relatively small sample and the self-reported characteristic of the measurements do not allow to draw conclusive conclusions about causality. A larger sample may heighten or decrease the differences between voice communication users and non-users. There could be confounding variables that the present paper dose not check such as personality characteristics (Collins \& Freeman, 2013) and motivation for play (Yee, 2006; Yang \& Liu, 2017). Finally, the current paper is not able to investigate the preexisting friendships, more precisely if these bounds that we find are formed through playing while using voice communication or voice chat is the result an already established bonds formed in game or otherwise.

In summary our results indicate that voice communication usage decreases perceived anonymity and increases two forms of Online Social Capital: Bonding and Bridging. Among the two forms of Online Social Capital, in-games voice communication seems to support slightly more the development of Online Bonding Social Capital. One explanation for our result is that voice communication influences Perceived Anonymity and individuals that consider themselves as having a low level of anonymity engage in more controlled behaviors that in turn facilitates the development of Social Capital.

\section{References}

Appel, L., Dadlani, P., Dwyer, M., Hampton, K., Kitzie, V., Matni, Z. A., . . Teodoro, R. (2014). Testing the validity of social capital measures in the study of information and communication technologies. Information, Communication \& Society, 17(4), 398-416. doi:10.1080/1369118X.2014.884612

Barlett, C. P., Gentile, D. A., \& Chew, C. (2016). Predicting Cyberbullying From Anonymity. Psychology of Popular Media Culture, 5(2), 171-180. doi:10.1037/ppm0000055 
Castiglione, D., Van Deth, J. W., \& Wolleb, G. (2008). The Handbook of Social Capital. Oxford University Press.

Chen, X., Sun, M., Wu, D., \& Song, X. Y. (2019). Information-Sharing Behavior on WeChat Moments: The Role of Anonymity, Familiarity, and Intrinsic Motivation. Frontiers in psychology, 10 , 2540. doi:10.3389/fpsyg.2019.02540

Cole, H., \& Griffiths, M. D. (2007). Social Interactions in Massively Multiplayer Online Role-Playing Gamers. CyberPsychology \& Behavior, 10(4), 575-583. doi:10.1089/cpb.2007.9988

Collins, E., \& Freeman, J. (2013). Do problematic and non-problematic video game players differ in extraversion, trait empathy, social capital and prosocial tendencies? Computers in Human Behavior, 29, 1933-1940. doi:10.1016/j.chb.2013.03.002

Curlew, A. E. (2019). Undisciplined Performativity: A Sociological Approach to Anonymity. Social Media + Society, 5(1). doi:10.1177/2056305119829843

Deleuze, J., Maurage, P., Schimmenti, A., Nuyens, F., Melzer, A., \& Billieux, J. (2019). Escaping reality through videogames is linked to an implicit preference for virtual over real-life stimuli. Journal of Affective Disorders, 245, 1024-1031. doi:10.1016/j.jad.2018.11.078

Dobrowolski, P., Hanusz, K., Sobczyk, B., Skorko, M., \& Wiatrow, A. (2015). Cognitive enhancement in video game players: The role of video game genre. Computers in Human Behavior, 44, 59-63. doi:10.1016/j.chb.2014.11.051

Ducheneaut, N., Yee, N., Nickell, E., \& Moore, R. (2007). The life and death of online gaming communities: a look at guilds in world of warcraft. Proceedings of the SIGCHI Conference on Human Factors in Computing Systems, (pp. 839-848).

Entertainment Software Association. (2019). 2019 Essential Facts About the Computer and Video Game Industry. Entertainment Software Association. doi:https:/www.theesa.com/wp-content/uploads/2019/05/ ESA_Essential_facts_2019_final.pdf

Granic, I., Lobel, A., \& Engels, R. R. (2014). The benefits of playing video games. American Psychologist, 69(1), 66-78. doi:10.1037/a0034857

Handrahan, M. (2019, May 13). Discord reaches 250 m users after just four years. (Gamer Network) Retrieved 2019, from gamesindustry.biz: https://www.gamesindustry.biz/articles/2019-05-13-discord-reaches-250musers-after-just-four-years

Hite, D. M., Voelker, T., \& Robertson, A. (2014). Measuring Perceived Anonymity: The Development of a Context Independent Instrument. Journal of Methods and Measurement in the Social Sciences, 5(1), 2239. doi:10.2458/v5i1.18305

Huvila, I., Holmberg, K., Ek, S., \& Widen, G. (2010). Social capital in Second Life. Online Information Review, 34(3), 295-316. doi:10.1108/14684521011037007

Interactive Software Federation of Europe. (2020). ISFE Key Facts 2020.

Kobayashi, T. (2010, September). Bridging Social Capital in Online Communities: Heterogeneity and Social Tolerance of Online Game Players in Japan. Human Communication Research, 36(4), 546 - 56. doi:10.1111/j.1468-2958.2010.01388.x

Krotoski, A. (2004). Chicks and joysticks: An exploration of women and gaming. Elspa (The Entertainment \& Leisure Sofware Publishers Association).

Lawrence, W. R., \& Grotz, J. (1977). The measurement of trust and its relationship to self-disclosure. Human Communication Research, 3(3 ), 250-257.

Lucas, K., \& Sherry, J. L. (2004). Sex differences in video game play: A communication-based explanation. Communication research, 31(5), 499-523. doi:10.1177/0093650204267930

Millen, D. R., \& Patterson, J. F. (2003). Identity disclosure and the creation of social capital. CHI'03 extended abstracts on Human factors in computing systems (pp. 720-721). Fort Lauderdale: Association for Computing Machinery. doi:10.1145/765891.765950

Molyneux, L., Vasudevan, K., \& Gil de Zúñiga, H. (2015, July). Gaming Social Capital: Exploring Civic Value in Multiplayer Video Games. Journal of Computer-Mediated Communication, 20(4), 381-399. doi:10.1111/jec4.12123

Nardi, B. (2010). My Life as a Night Elf Priest: An Anthropological Account of World of Warcraft. University of Michigan Press. 
Newzoo. (2021). Games, esports, and mobile trends to watch in 2021. Newzoo. Retrieved from https://resources.newzoo.com/hubfs/Reports/2021_Newzoo_Trends_to_Watch_Report.pdf?utm_campaign=Global\%20Mobile\%20Market\%20Report\%202020\&utm_medium $=$ email\&_hsmi $=113081565 \&$ hsenc $=$ p2AN qtz-8hPKQmcWXX2nySRuGTjctrFWquwgpGhC8tEWUR6EMRJdhP17O1mnGgDhzDglxritAFmPZCb

Putnam, R. D. (2000). Bowling Alone: The Collapse and Revival of American Community. New York, United States: Simon \& Schuster.

Sharon, T., \& John, N. A. (2018). Unpacking (the) secret: Anonymous social media and the impossibility of networked anonymity. New Media \& Society, 20(11), 4177-4194. doi:10.1177/1461444818768547

Steinkuehler, C., \& Duncan, S. (2008, December). Scientific Habits of Mind in Virtual Worlds. Journal of Science Education and Technology, 17(6), 530-543. doi:10.1007/s10956-008-9120-8

Steinkuehler, C., \& Williams, D. (2006). Where Everybody Knows Your (Screen) Name: Online Games as “Third Places". Journal of Computer-Mediated Communication, 11(4), 885-909. Retrieved 3 17, 2019, from https://academic.oup.com/jcmc/article/11/4/885/4617703

Sticca, F., \& Perren, S. (2013). Is cyberbullying worse than traditional bullying? Examining the differential roles of medium, publicity, and anonymity for the perceived severity of bullying. Journal of youth and adolescence, 42(5), 739-750. doi:10.1007/s10964-012-9867-3

Trepte, S., Reinecke, L., \& Juechems, K. (2012). The social side of gaming: How playing online computer games creates online online and offline social support. Computers in Human Behavior, 28, 832-839. doi:10.1016/j.chb.2011.12.003

Tsikerdekis, M. (2013). The effects of perceived anonymity and anonymity states on conformity and groupthink in online communities: A W ikipedia study. Journal of the American Society for Information Science and Technology, 64(5), 1001-1015. doi:10.1002/asi.22795

Vella, K., Johnson, D., \& Hides, L. (2013, October). Positively playful: When videogames lead to player wellbeing. In Proceedings of the First International Conference on Gameful Design, Research, and Applications (pp. 99-102). Stratford, Ontario, Canada: ACM.

von der Heiden, J. M., Braun, B., Muller, K. W., \& Egloff, B. (2019). The Association Between Video Gaming and Psychological Functioning. frontiers in Psychology. doi:10.3389/fpsyg.2019.01731

Wadley, G., Gibbs, M., Hew, K., \& Graham, C. (2003). Computer Supported Cooperative Play, "Third Places" and Online Videogames. In S. Viller, \& P. Wyeth (Ed.), Proceedings of the Thirteenth Australian Conference on Computer Human Interaction (OzChi 03) (pp. 238-241). Brisbane: University of Queensland.

Williams, D. (2006). On and Off the Net: Scales for Social Capital in an Online Era. Journal of ComuterMediated Communication, 593-628. doi:10.1111/j.1083-6101.2006.00029.x

Williams, D., Caplan, S., \& Xiong, L. (2007). Can You Hear Me Now? The Impact of Voice in an Online Gaming Community. Human Communication Research, 33(4), 427-449. doi:10.1111/j.14682958.2007.00306.x

Williams, D., Ducheneaut, N., Xiong, L., Zhang, Y., Yee, N., \& Nickell, E. (2006). From Tree House to Barracks: The Social Life of Guilds in World of Warcraft. Games and Culture, 1(4), 338-361. Retrieved 3 17, 2019, from http://nickyee.com/pubs/tree house to barracks 2006.pdf

Yang, C.-c., \& Liu, D. (2017). Motives Matter: Motives for Playing Pokémon Go and Implications for WellBeing. Cyberpsychology, Behavior, and Social Networking, 20(1), 52-57. doi:10.1089/cyber.2016.0562

Yee, N. (2006). Motivations for play in online games. CyberPsyhology \& Behavior, 9(6).

Zhai, Y. (2019). The role of online social capital in the relationship between Internet use and self-worth. Current Psychology, 1-10. doi:10.1007/s12144-019-0149-z

Zhong, Z. J. (2011). The effects of collective MMORPG (Massively Multiplayer Online Role-PlayingGames) play on gamers' online and offline social capital. Computers in Human Behavio, 27, 2352-2363. doi:10.1016/j.chb.2011.07.014. 